\title{
SARS-COV-2. Origen, vacunas, variantes y el futuro de la pandemia
}

\section{SARS-COV-2. Origin, vaccines, variants and the future of the pandemic}

\author{
AdRIANA DELFRARo \\ Sección Virología. Facultad de Ciencias. Universidad de la República. Montevideo, Uruguay. \\ ORCID. 0000-0003-3701-9621 \\ adriana@fcien.edu.uy
}

\section{Resumen}

Desde fines de 2019 estamos viviendo un evento pandémico único en la historia de la humanidad. El antecedente más cercano, por su duración y sus consecuencias fue la pandemia de la gripe de 1918. A pesar de las múltiples consecuencias de este evento para salud pública, hoy nos encontramos mucho mejor preparados para combatir esta virosis y tratar a los pacientes con COVID-19. Conocimos rápidamente la identidad y las principales características del agente etiológico y apoco más de un año de declarada la emergencia sanitaria se han desarrollado vacunas que vienen mostrando ser efectivas y seguras. El objetivo de este trabajo es repasar las principales características del virus SARS-COV-2ylas diferencias y similitudes con otros coronavirus relacionados. Revisaremos los tipos y el desempeño de las vacunas actualmente en uso, y los desafíos que enfrentamos ante el surgimiento de ciertas variantes virales.

Palabras clave: virus, SARS-COV-2, vacunas, variabilidad.

\section{Abstract}

Since the end of 2019 we are living a unique pandemic event in the human history. The closest precedent, for its lasting and consequences was the 1918 influenza pandemic. Despite the multiple consequences for public health, we are now much better prepared to fight this agent and to provide a better treatment to COVID-19 patients. The scienti- fic community discovered soon the identity and the key biologic characteristics of the COVID-19 etiologic agent, and just over a year and a half from the pandemic declaration, safe and effective vaccines were developed. In the present work, we aim to look over the main characteristics of SARS-CoV-2, as well as the differences and similarities with related coronaviruses. We will review the different vaccine platforms and the performances of the vaccines in use and discuss the future challenges of the emergence of viral variants.

Keywords: virus, SAR-COV2, vaccines, variability.

\section{Generalidades del virus}

Los coronavirus pertenecen al orden Nido virales, subfamilia Coronavirinae, y se dividen en 3 subgéneros: Alfa, Beta y Gamma coronavirus. Se encuentran entre los virus ARN con mayor tamaño genómico e infectan aves y mamíferos (porcinos, bovinos, felinos, roedores, camélidos, primates). En el hombre, son agentes causales de infecciones respiratorias leves y graves. En años recientes, se han encontrado numerosos coronavirus asociados a murciélagos, los cuales son considerados como reservorios y fuente potencial de evolución y dispersión de estos virus (Fields B., 2013).

EI SARS-COV-2pertenece al género Betacoronavirus, subgénero Sarbecovirus. Estructuralmente es un virus envuelto, y su genoma es una molécula de ARN de cadena simple y polaridad positiva de aproximadamente 30 kilobases (kb). Dentro del género, sus parientes más cercanos son el SARS-1 y varios coronavirus específicos 
de murciélagos. Algo más divergentes se encuentran el virus MERS (Middle East Respiratory Syndrome) y los coronavirus humanos estacionales HCov-NL63, 229E, HKU1 y OC43 (Monto et al., 2020).

Los coronavirus poseen un ciclo de multiplicación que involucra en primer lugar la entrada a la célula, por fusión directa de la envoltura viral con la membrana celular(como es el caso de SARS-COV-2), o bien a través de endocitosis mediada por receptores (ej. SARS1 y HCov229E). Para esto hacen uso de la proteína S (espiga o "spike" por su denominación en inglés) en el virus y moléculas presentes en la superficie de la célula que actúan como receptor. En particular, el SARSCOV-2 utiliza el receptor de la enzima convertidora de angiotensina 2 (ACE2), que interactúa con la región RBD ("receptor-bindingdomain") de S. La síntesis de proteínas virales involucra la transcripción y traducción directa de la mayor parte del genoma en proteínas, en tanto una zona adyacente al extremo3' del genoma se transcribe como ARNs mensajeros subgenómicos (figura 1) (Maier et al., 2015).
Como característica compartida con todos los virus ARN, el SARS-COV-2 presenta una importante variabilidad genética. Las ARN polimerasas en general no son capaces de corregir errores en el proceso de replicación, introduciendo un número importante de mutaciones en cada ronda de replicación. Sin embargo, se ha observado que los coronavirus, entre otros miembros del orden Nidovirales, poseen una ARN polimerasa con cierta capacidad de corrección de errores, por lo que presentan menores tasas de mutación si los comparamos con otros virus humanos como el de la gripe o el virus de la inmunodeficiencia humana (VIH).Los coronavirus cuentan con otra fuente importante para generar diversidad genética: su capacidad de recombinación. Desde el punto de vista evolutivo, estas características les han permitido mantener una funcionalidad compatible con el gran tamaño genómico y lo complejo de su ciclo de multiplicación, y a su vez una diversidad genética que les confiere una gran capacidad deadaptación al hospedero (Gorbalenya et al., 2006).

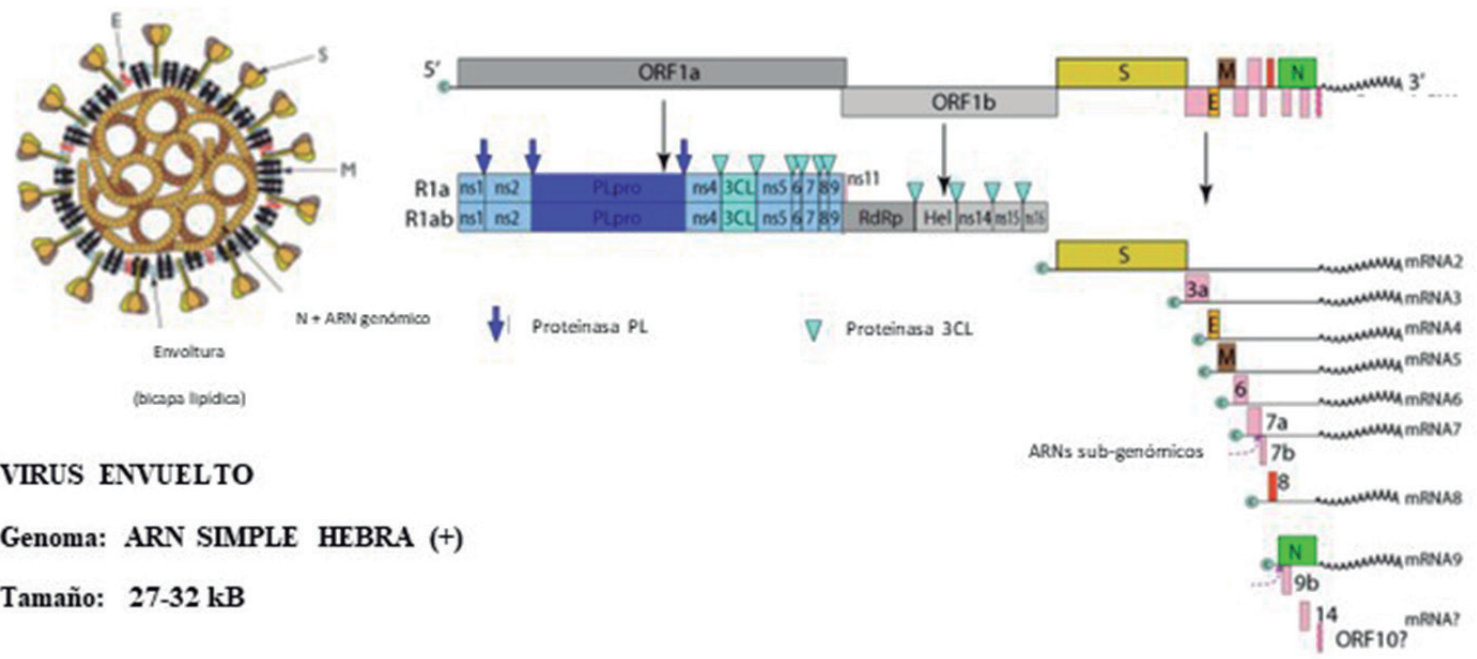

\section{Proteínas estructurales}

E: proteina pequeña de la envoltura

S: espiga (glicoproteina)

M: proteina de membrana

$\mathrm{N}$ : nucleoproteina

Figura 1. Esquema general de la partícula viral de un coronavirus y su organización genómica. Modificado de ViralZone: https://viralzone.expasy.org/ 


\section{Emergencia de SARS y MERS. Un cambio de paradigma}

Hasta el año 2002, los coronavirus humanos conocidos eran agentes causales de infecciones respiratorias leves. La aparición de un brote de una enfermedad respiratoria grave en China, no atribuible a los patógenos respiratorios conocidos en ese momento, dio lugar a un cambio radical en la historia de estos virus. El primer caso de esta nueva entidad clínica fue reportado en la provincia de Guandong (China), el 16 de noviembre de 2002. Fueron necesarios varios meses de investigación y un esfuerzo colaborativo internacional para demostrar que el agente etiológico era un nuevo coronavirus. Inicialmente fue denominado virus Urbani, en homenaje a Carlo Urbani, médico italiano que reconoció por primera vez la nueva entidad clínica y que falleció a causa de ésta. Posteriormente, este agente viral fue denominado virus del SARS (Severe Acute Respiratory Syndrome, por sus siglas en inglés). En julio de 2003 la OMS declara el final de la epidemia, con un total de 8000 casos confirmados y 774 fallecidos. De estos, 2700 casos y 425 fallecidos ocurrieron fuera de China (otros países de Asia, Europa y Norteamérica). La trasmisión de este virus se da a través de pacientes que se encuentran en la fase sintomática de la enfermedad, la mayoría de los casos ocurren en el ambiente intrahospitalario, lo que permitió en su momento una contención relativamente eficaz de la epidemia. El 5 de julio de 2006 la OMS declara el fin de la epidemia, y a partir de esa fecha no se han registrado nuevos casos. Años más tarde fue posible identificar el ciclo natural de este virus, que involucra a los murciélagos del género Rhinolophussp como reservorio natural y a la civeta ( $\mathrm{Pa}$ gumalarvata), un carnívoro de la familia Viverridae, como hospedador intermedio (Ksiazek et al., 2003; Lau et al., 2005; Banos and Lacasa, 2007).

El segundo hito en la historia de estos virus fue la emergencia de una enfermedad respiratoria aguda denominada MERS: Middle East Respiratory Syndrome, y la identificación de su agente etiológico. Nuevamente un coronavirus, en este caso distinto al SARS, pero relacionado, era capaz de provocar una enfermedad respiratoria grave, con una elevada letalidad, que alcanzó el $35 \%$ de los casos. El primer caso de esta virosis fue identificado en junio 2012, en Jeddah, Arabia Saudita. Posteriormente se expandió por 27 países, mayoritariamente del Medio Oriente, alcanzando a Corea del Sur. Los últimos datos de 2019 reportan 2260 casos confirmados y 803 fallecidos. Este virus tiene como reservorio a los murciélagos de las especies Taphozous- perforatus, Rhinopomahardwickii, Pipistrelluskuhlii, y su hospedador intermedio son los camélidos (dromedarios). El modo de trasmisión es diferente al SARS y a lo que conocemos del SARS-COV-2, la trasmisión mayormente ocurre desde los camélidos, y en menor medida a partir de casos intrahospitalarios o de convivientes. También se ha observado que posee una tasa de trasmisión menor, con un número de reproducción básico (R0) de entre 0,5-0,7 (Memish et al., 2013; Cui et al., 2019; Song et al., 2019).

Estos hallazgos fueron claves para avanzar en el estudio de los ciclos naturales de estos virus, y cada vez fue más evidente la diversidad de este grupo y la asociación de varios de ellos a los murciélagos como reservorios naturales. Fue así como se determinó que los coronavirus humanos HCoV-NL63 y HCoV-229E tenían como reservorio a diferentes especies de quirópteros y se identificó una importante variedad de coronavirus no patogénicos para el hombre, que son exclusivos de murciélagos (Li et al., 2005; Anthony et al., 2013; Cui et al., 2019).

\section{SARS-COV-2. El origen y la expansión de un nuevo coronavirus}

En 2013, Smith y Wang nos alertaban: "con la actividad humana solapándose cada vez más a los hábitats de los murciélagos, no hay duda de que los virus zoonóticos continuarán emergiendo desde estos reservorios. Para ayudar a predecir y prevenir la emergencia viral, necesitamos un mayor entendimiento de las dinámicas de infección en los hospedadores y entender el impacto de los cambios en el ambiente ocasionados por el hombre sobre el potencial de los virus para lograr "saltos de especie". Se requiere un enfoque integrado de "Una Salud", con la participación de científicos internacionales, ecologistas, veterinarios, profesionales de la salud, cientistas sociales y políticos, trabajando en conjunto para minimizar el impacto de las enfermedades zoonóticas asociadas a murciélagos. Es critico que logremos coexistir con los murciélagos, dado que estas singulares criaturas son vitales para nuestros ecosistemas" (Smith and Wang, 2013).

Algunos años después, ocurrirá la "tormenta perfecta". En 2018, ya se habían reportado evidencias de contacto humano con coronavirus de murciélagos, a través de la detección de anticuerpos en poblaciones de la provincia de Yunnan, China, cercanas a cuevas donde habitan estos mamíferos (Wang et al., 2018). En diciembre de 
2019 aparecen los primeros casos de neumonía severa en Wuhan (China), inicialmente asociados a un mercado de animales vivos típico de la zona. La enfermedad se denominó COVID-19, y la historia que sigue es muy conocida por todos. Gracias al desarrollo y el mayor acceso a tecnologías como la secuenciación de nueva generación (NGS por su sigla en inglés) y a un enorme esfuerzo colaborativo internacional, en enero de 2020 ya conocíamos la secuencia genómica completa de este nuevo virus y teníamos una fuerte evidencia de su origen natural: el virus filogenéticamente más emparentado con SARS-COV-2 es un coronavirus de murciélago identificado en un ejemplar de la especie Rinolophusaffinis, capturado en 2013 en la provincia de Yunnan (Andersen et al., 2020).

Hoy en día, la información virológica, inmunológica, clínica y epidemiológica aumenta de forma exponencial cada día y la velocidad a la que vamos conociendo la biología de este agente infeccioso desde sus varias aristas es avasallante.

Las características biológicas del virus, su modo de trasmisión, así como factores biológicos, sociales y comportamentales del hombre, han provocado una rápida expansión y ha sido sumamente complejo controlar el avance de esta virosis, que tomó rápidamente el carácter de pandemia. Se cree que este virus ha ingresado recientemente a la población humana (en términos evolutivos), por lo que tiene a disposición una gran masa de hospederos sin "experiencia "previa desde el punto de vista inmune, favoreciendo por tanto una experiencia "previa diferencia de sus parientes SARS y MERS, este virus se trasmite eficientemente en pacientes asintomáticos y también en la etapa presintomática. Se suman además la ocurrencia de eventos de "super dispersión", donde algunos individuos son capaces de excretar gran cantidad de virus por tiempo prolongado, generando así un número importante de contagios (Andersen et al., 2020).

La elevada circulación viral, como se constata ahora mismo en Uruguay y en otros países de Latinoamérica, genera un ambiente propicio para el surgimiento de variantes, algunas con mayor capacidad de trasmisión y por tanto capaces de sustituir a los virus parentales, con menor "fitness" relativo. La resistencia a antivirales o la emergencia de variantes que escapan a la respuesta inmune natural o inducida por vacunas, son fenómenos ampliamente conocidos en virología como mecanismos moduladores de la diversidad y la evolución de los virus ARN.
En este marco, una de las intervenciones de salud pública que se impone para contribuir al control de esta virosis es el desarrollo de vacunas seguras y eficaces.

¿Como surgieron los candidatos vacunales?, ¿Como se llegó en menos de un año a la fabricación las intervenciones vacunas contra el SARS-COV-2?

\section{Vacunas virales, tipos y fases de desarrollo}

Según la definición de OMS, una vacuna es "cualquier preparación destinada a generar inmunidad contra una enfermedad estimulando la producción de anticuerpos. Puede tratarse, por ejemplo, de una suspensión de microorganismos muertos o atenuados, o de productos o derivados de microorganismos". Hacia finales del siglo XX la esperanza de vida del hombre se extendió 30 años. En esto incidió el acceso a agua potable una mejor alimentación, junto con importantes progresos en la atención médica donde los antibióticos y el desarrollo de vacunas fueron primordiales.

Las vacunas virales más tradicionales son las vacunas atenuadas e inactivadas. En una vacuna atenuada, el virus es "debilitado" en su patogenicidad por sucesivos pasajes en cultivos de células u otro junto con, por tanto, al administrar la vacuna ocurre infección, pero no enfermedad. La vacuna contra la fiebre amarilla pertenece a esta categoría.

Las vacunas inactivadas se generan también a partir del virus completo, el cual es inactivado por un agente químico (ej.: beta-propio lactona). En este caso, el virus no es capaz de infectar ni provocar enfermedad, pero se presenta con sus principales determinantes antigénicos intactos (ej.: las proteínas de superficie) ante el sistema inmune. La vacuna antipoliomielítica Salk es un ejemplo de vacuna a virus inactivado. Generalmente las vacunas vienen formuladas con compuestos adyuvantes, que aumentan la respuesta inmune, además de conservantes o preservantes.

En los últimos años se han desarrollado vacunas con diferentes tecnologías: utilizando únicamente las proteínas del virus (vacunas a subunidades proteicas como la del virus hepatitis $B$ ), vacunas a vectores virales (vacuna contra el virus Ébola) y vacunas basadas en ADN o ARN.

El desarrollo de una vacuna hasta su producción en masa y aplicación a escala poblacional debe pasar por un proceso generalmente largo, que implica varias etapas, denominadas fases (figura 2). 


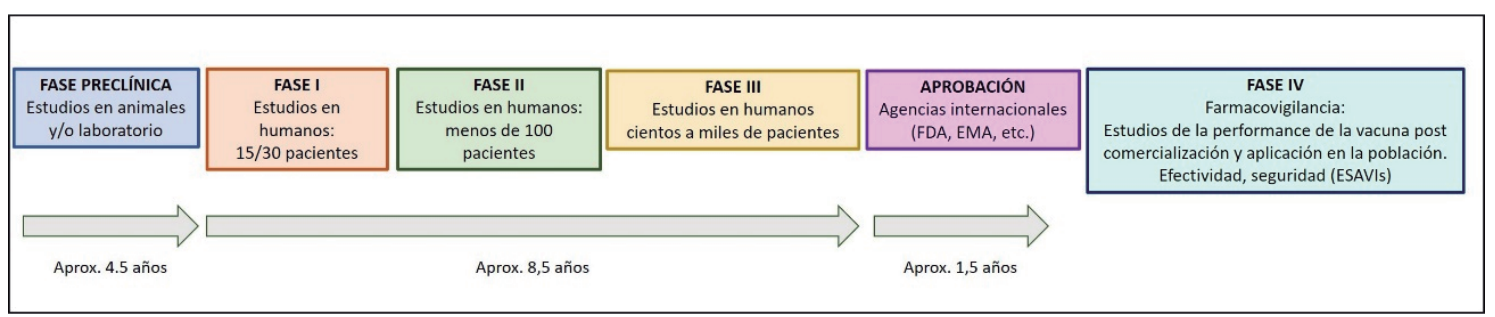

Figura 2. Esquema de las etapas en el desarrollo de un candidato vacunal.

La fase preclínica implica el diseño y elaboración a pequeña escala de la vacuna, así como las primeras pruebas midiendo su eficacia y seguridad en base a experimentos controlados en animales de laboratorio. Una vez que se muestra que la vacuna candidata es inmunogénica y posee un perfil aceptable de seguridad, se pasa a las fases siguientes, donde se ensaya en el destinatario final (humano o animal), en pocos individuos sanos (fase II). Normalmente, en esta fase se analizan diferentes dosis, son estudios controlados y canonizados donde, para el caso de vacunas de uso humano se canonizados con diferentes estados de salud (comorbilidades) y diversos grupos demográficos. Los estudios fase III involucran cientos de miles de individuos, también son estudios controlados y canonizados e involucran grupos demográficos más amplios, a fin de que se represéntelo más fielmente la diversidad poblacional que recibirá a futuro la vacuna. De estos ensayos se obtiene ensayos se a la respuesta inmune generada por la vacuna, eficacia y seguridad. En la web: https:// clinicaltrials.gov/ct2/home se encuentran inscriptos y se puede acceder a la información sobre los ensayos clínicos, (sea con financiamiento público o privado) que se están desarrollando a nivel mundial, entre ellos, podemos explorar los relacionados con SARS-COV-2 y Covid-19 (vacunas, terapéutica, antivirales, etcétera).

Luego de los ensayos fase III y previo a su aplicación, se requiérela autorización de las agencias reguladoras, entre las más conocidas internacionalmente mencionamos la FDA (Food and Drug Administration, USA) y la EMA (European Medicines Agency). En situaciones de emergencia sanitaria, es posible, en base al análisis de los resultados de fase III por parte de grupos de especialistas, otorgar una aprobación de emergencia previa a la aprobación final. Los países tienen además sus propias agencias reguladoras (por ejemplo, ANMAT en Argentina, ANVISA en Brasil) (Polack et al., 2020; Sadoff et al., 2021; Xia et al., 2021).

Una vez aprobadas, se continúa con la fase IV, denominada fármaco vigilancia. En esta etapa post-comercialización, se realiza una vigilancia estrecha de posibles efectos adversos, los Ilamados ESAVIs (Eventos Supuestamente Atribuibles a Vacunación e Inmunización).

\section{Vacunas contra SARS-COV-2}

El desarrollo de las vacunas contra este virus pandémico fue rápido. A junio de 2020 ya había más de 120 candidatos en fase preclínica y unos 13 en fase clínica. En marzo-abril de 2021 se contaba con 273 vacunas en desarrollo, de las cuales 87 se encontraban en fases preclínicas, 23 en fase II-III y 4 habían pasado a fase Icono ocurre frecuentemente en el camino de obtener una vacuna para cualquier patógeno, un número importante de candidatos no alcanza los niveles requeridos de eficacia y/o seguridad y no spasan las fases experimentales. El requerimiento de OMS es que una vacuna debe tener una eficacia mínima del 50\%.

Podemos decir que la actual pandemia es la mayor experimentada por la humanidad, en extensión y morbi-mortalidad, después de la pandemia de influenza H1N1 de 1918. La enorme diferencia es que ahora contamos con el conocimiento para caracterizar rápidamente el tipo de patógeno involucrado y sus características y contamos con la tecnología para formular vacunas eficaces y seguras. Para este virus, varios elementos ayudaron al rápido desarrollo de las vacunas. Entre estos, podemos mencionarlas experiencias previas con virus relacionados como SARS y MERS, tanto en el conocimiento del agente infeccioso como en la formulación de vacunas contra ellos. A esto se ha sumado una fuerte inversión tanto pública como privada. También se aceleraron ciertos procesos burocráticos en varios ensayos las fases se realizaron de manera conjunta (Fase I/II o II/III). La elevada circulación viral también contribuyó a que los ensayos clínicos que requieren el reclutamiento de cientos de miles de voluntarios (faselli) se hiciera rápidamente, muchas veces en más de un país de forma paralela. Esto puede llevar un tiempo considerablemente mayor cuando se trata de enfermedades menos prevalentes o de circulación muy localizada. 
Las principales vacunas disponibles al momento pertenecen a 4grandes grupos:

\section{- Inactivadas:}

Basadas en el virus completo inactivado. Se trata de un sistema conocido y probado, generalmente con muy pocos efectos adversos, aunque requieren mayor tiempo para su producción reformulación. Ej: Coronavac (Sinovac), Sinopharm (Xia et al., 2021; Zhang et al., 2021). Estas vacunas son altamente eficaces en prevenir enfermedad grave y muerte (85 a 97\%), y alcanzan una eficacia que ronda el $60 \%$ para prevenir la enfermedad sintomática.

- Basadas envectoradenovirus: Oxford- AstraZeneca, Janssen, Sputnik V.

Se basan en la expresión de la proteína de interés, a través de su inclusión en un Adenovirus deficiente en replicación, que actúa como vector. Como resultado, se sintetiza una proteína $S$ "optimizada" en el uso de codones, induciendo respuesta inmune protectora tanto humoral como celular. Las diferentes plataformas utilizan diferentes vectores adenovirales humanos (adenovirus tipo 5 y adenovirustipo 26 en el caso de Sputnik $V$, adenovirus tipo 26, Janssen) o de primates (ChadOx, vacuna Astra Zeneca) (Folegatti et al., 2020; Logunov et al., 2021). Estas tecnologías ya se habían utilizado para vacunas contra virus Ébola. Se observa una eficacia elevada, tanto para enfermedad grave y letalidad, como para enfermedad sintomática (entre $82 \%$ y $90 \%$ ). Durante la farmacovigilancia de estas vacunas, sehan reportado y se encuentran en estudio algunos eventos de trombosis venosa presumiblemente asociados a la vacuna de Astra Zeneca, los que ocurrieron mayoritariamente en mujeres menores de 55 años. En nuestro país, en base a los datos disponibles y a la evaluación beneficio-riesgo, se ha indicado su administración a mayores de 60 años.

- Basadas em ARNm: Comirnaty (Pfizer), mRNA1273 (Moderna)

Se basan em una molécula de ARN mensajero que codifica la proteína S. El ARN, que en el caso de "Comirnaty" contiene nucleósidos modificados para mejorar su estabilidad, es liberado en la célula y se utiliza como molde para sintetizar la proteína. En el diseño de la vacuna de Moderna, S se sintetizaren su configuración pre-fusión, lo que mejora su inmunogenicidad. La proteína sintetizada será reconocida entonces por el sistema inmune, generando una respuesta humoral y celular con capacidad neutralizante y protectora (Polack et al., 2020; Baden et al., 2021). Esta vacuna ha demostrado alta eficacia en los ensayos clínicos, y alta efectividad también a nivel poblacional, tanto para prevenir casos graves (90 a 100\% según los ensayos) como para enfermedad sintomática (95\%), demostrando también eficacia en disminuir las infecciones asintomáticas (entre 60 y 90\%) (Amit et al., 2021; Dagan et al., 2021; DIGESA, MSP, 2021). En los ensayos clínicos mostró un perfil de seguridad adecuado, reportándose episodios de fiebre, dolor en el sitio de inyección, cansancio, más frecuentes en los voluntarios jóvenes. Los efectos adversos severos tuvieron baja incidencia, sin diferencias significativas entre los que recibieron la vacuna y los que recibieron placebo (Polack et al., 2020; Dagan et al., 2021). En la farmacovigilancia no se ha informado hasta el momento de eventos adversos severos.

- Vacunas a subunidades proteicas: Ej. Novavax, Soberana, Abdala (estas últimas, en trámite de aprobación por OMS).

En este caso se produce la proteína a gran escala (ej.: a través un sistema de expresión como el de baculovirus o levaduras) y se administra vía nanopartículas, junto con un adyuvante. Hay experiencias previas de vacunas virales actualmente en uso que se basan en la misma tecnología, por ejemplo, la de papilomavirus (HPV) o hepatitis B (HBV) (Keech et al., 2020). De acuerdo con lo anunciado por el fabricante, en base a los resultados de los ensayos fase III, la vacuna Novavax mostró $100 \%$ de protección contra enfermedad moderada y severa y $90,4 \%$ de eficacia global(datos no publicados).

\section{Variantes del SARS-COV-2}

Como nunca en la historia, la variabilidad genética de este virus se conoce casi en tiempo real, gracias a la potente herramienta de la secuenciación NGS. Esto ha permitido obtener un número muy importante y una amplia representación geográfica de las secuencias genómicas completas del virus. Esta información ha venido creciendo de forma exponencial a tan solo un año y medio de su emergencia: a junio 2021, hay más de 2 millones de secuencias depositadas en repositorios públicos como GISAID o GenBank, y el número crece día a día.

Esto ha permitido un conocimiento pormenorizado de los cambios genómicos que el virus va adquiriendo 
a medida que circula en la población, y por tanto el monitoreo de la emergencia de variantes virales. Las variantes son virus con ciertas mutaciones, $u$ otros cambios en su genoma como deleciones o inserciones, eventos que constituyen un fenómeno natural y esperable en los virus ARN. Entre estos, son de especial interés las mutaciones que tienen lugar en proteínas clave del virus, como la proteína de superficie $S$. En particular, es importante conocer y monitorear las mutaciones en la región RBD (región de unión al receptor) de esta proteína viral, ya que pueden afectar zonas (epítopos) relevantes para la neutralización por anticuerpos, o pueden afectan la afinidad de unión del virus a la célula huésped.

Esta información es dinámica y en base a los hallazgos de secuencias genómicas, la epidemiología y los ensayos de laboratorio, la OMS ha puesto especial atención y ha definido las denominadas variantes de interés (VOIs) y variantes de preocupación (VOCs). Estas poseen ciertas mutaciones o muestran alguna evidencia en su comportamiento biológico que hace necesario su monitoreo. Algunas de estas características son su mayor trasmisibilidad y por ende su rápida diseminación, o la capacidad de evadir parcialmente la neutralización por vacunas y/o sueros de pacientes convalecientes.

Definiciones OMS: Un aislado de SARS-CoV-2 es una variante de interés (VOI) si, en comparación con un aislado de referencia, su genoma tiene mutaciones con implicaciones fenotípicas establecidas o presuntas, y, o bien:

1. Ha sido identificada como causa de transmisión comunitaria o de múltiples casos/grupos de COVID-19, o ha sido detectada en múltiples países; O BIEN

2. La OMS, en consulta con el Grupo de trabajo de la OMS sobre la evolución del virus SARS-CoV-2, ha determinado que se trata de una VOI.

Variante de preocupación (VOC): Una variante del SARS-CoV-2 que cumple con los criterios para ser definida como una VOI en relación con la cual se ha demostrado, tras una evaluación comparativa, que está asociada a uno o más de los siguientes cambios en un grado que resulte significativo para la salud pública mundial:

3. Aumento de la transmisibilidad o cambio perjudicial en la epidemiología de la COVID-19, o

4. Aumento de la virulencia o cambio en la presentación clínica de la enfermedad; o
5. Disminución de la eficacia de las medidas sociales y de salud pública o de los medios de diagnóstico, las vacunas y los tratamientos disponibles.

Estas variantes, especialmente las "de preocupación" nos colocan ante un desafío importante. Algunas han demostrado alta trasmisibilidad, pasando a dominar rápidamente cuando ingresan y se dispersan en un país o región (ej. la VOCGamma, dominante actualmente en Uruguay y en varios países de Sudamérica, o la VOC Delta en India, Estados Unidos, Reino Unido). Ciertas variantes muestran además que son menos neutralizables por los anticuerpos vacunales, o por los anticuerpos generados por la infección natural con virus heterólogos, lo que puede dar lugar a reinfecciones (Andrade et al., 2021; Naveca et al., 2021; Palacios et al., 2021; Xie et al., 2021). Es posible que, en un futuro, del mismo modo que ocurre con la vacuna antigripal, sea necesario reformular o general vacunas polivalentes, y que debamos dar refuerzos periódicos.

Es importante pensar fenómenos como este evento pandémico a escala global. En un contexto de alta circulación viral, es virtualmente imposible detener el ingreso y dispersión de nuevas variantes una vez que aparecen en escena, en especial aquellas con mayor trasmisibilidad, por lo cual la vigilancia genómica y epidemiológica es altamente relevante. Es indispensable inmunizar rápidamente a las poblaciones e intentar bajar al máximo la circulación viral con medidas no farmacológicas, acompañando el proceso de vacunación. Las áreas geográficas con alta circulación y bajo nivel de vacunación, y la presencia de grandes grupos poblacionales susceptibles, son terreno fértil para el surgimiento de variantes. Existe una gran desigualdad a nivel mundial en cuanto al acceso y distribución a las vacunas, por lo que se hace necesario un esfuerzo colectivo y global, más allá de fronteras políticas, económicas y religiosas para combatir primordialmente los efectos más duros de la pandemia, como su letalidad. Hoy sabemos que además la enfermedad COVID-19 puede dejar secuelas importantes (Nalbandian et al., 2021), por lo que disminuir al máximo la morbilidad por este virus también es un objetivo muy importante por lograr en un futuro inmediato. En este sentido, las vacunas están entre las mejores herramientas de salud pública con las que contamos para avanzar hacia estos objetivos. 
VOIs (variantes de interés) a junio 2021

\begin{tabular}{|c|c|c|c|c|c|}
\hline $\begin{array}{l}\text { Denominación } \\
\text { de la OMS }\end{array}$ & $\begin{array}{l}\text { Linaje Pango } \\
\text { lineage }\end{array}$ & $\begin{array}{l}\text { Clado/linaje } \\
\text { GISAID }\end{array}$ & $\begin{array}{l}\text { Clado } \\
\text { Nextstrain }\end{array}$ & $\begin{array}{l}\text { Primeras muestras } \\
\text { documentadas }\end{array}$ & $\begin{array}{l}\text { Fecha de } \\
\text { designación }\end{array}$ \\
\hline Épsilon & B.1.427/B.1.429 & $\mathrm{GH} / 452 \mathrm{R} . \mathrm{V} 1$ & $21 \mathrm{C}$ & $\begin{array}{l}\text { Estados Unidos de } \\
\text { América, marzo } \\
2020\end{array}$ & 5 marzo 2021 \\
\hline Zeta & P.2 & GR/484K.V2 & $20 \mathrm{~B} / \mathrm{S} .484 \mathrm{~K}$ & Brasil, abril 2020 & 17 marzo 2021 \\
\hline Eta & B.1.525 & G/484K.V3 & 21D & $\begin{array}{l}\text { Múltiples países, } \\
\text { diciembre } 2020\end{array}$ & 17 marzo 2021 \\
\hline Theta & P.3 & GR/1092K.V1 & $21 \mathrm{E}$ & $\begin{array}{l}\text { Filipinas, enero } \\
2021\end{array}$ & 24 marzo 2021 \\
\hline lota & B.1.526 & $\mathrm{GH} / 253 \mathrm{G} . \mathrm{V} 1$ & $21 \mathrm{~F}$ & $\begin{array}{l}\text { Estados Unidos de } \\
\text { América, noviembre } \\
2020\end{array}$ & 24 marzo 2021 \\
\hline Kappa & B.1.617.1 & G/452R.V3 & 21B & India, octubre 2020 & 4 abril 2021 \\
\hline Lambda & C. 37 & GR/452Q.V1 & $20 D$ & Perú, Agosto 2020 & 14 junio 2021 \\
\hline
\end{tabular}

VOCs (variantes de preocupación) a junio 2021

\begin{tabular}{|c|c|c|c|c|c|}
\hline $\begin{array}{l}\text { Denominación } \\
\text { de la OMS }\end{array}$ & $\begin{array}{l}\text { Linaje } \\
\text { Pango } \\
\text { lineage }\end{array}$ & $\begin{array}{l}\text { Clado/linaje } \\
\text { GISAID }\end{array}$ & $\begin{array}{l}\text { Clado } \\
\text { Nextstrain }\end{array}$ & $\begin{array}{l}\text { Primeras muestras } \\
\text { documentadas }\end{array}$ & $\begin{array}{l}\text { Fecha de } \\
\text { designación }\end{array}$ \\
\hline Alpha & B.1.1.7 & $\begin{array}{l}\text { GRY(anteriormente } \\
\text { GR/501Y.V1) }\end{array}$ & 201 (V1) & $\begin{array}{l}\text { Reino Unido, } \\
\text { septiembre } 2020\end{array}$ & 18 diciembre 2020 \\
\hline Beta & B.1.351 & GH/501Y.V2 & $20 \mathrm{H}$ (V2) & $\begin{array}{l}\text { Sudáfrica, } \\
\text { mayo } 2020\end{array}$ & 18 diciembre 2020 \\
\hline Gamma & P.1 & GR/501Y.V3 & $20 \mathrm{~J}(\mathrm{~V} 3)$ & $\begin{array}{l}\text { Brasil, } \\
\text { noviembre } 2020\end{array}$ & 11 enero 2021 \\
\hline Delta & B.1.617.2 & G/478K.V1 & $21 \mathrm{~A}$ & $\begin{array}{l}\text { India, } \\
\text { octubre } 2020\end{array}$ & $\begin{array}{l}\text { VOI: } 4 \text { abril } 2021 \\
\text { VOC: } 11 \text { mayo } \\
2021\end{array}$ \\
\hline
\end{tabular}

Figura 3. Variantes de interés (VOIs) y variantes de preocupación (VOCs) de acuerdo a la clasificación de OMS: https://www. who.int/es/activities/tracking-SARS-CoV-2-variants 


\section{Referencias}

Amit, S., Regev-Yochay, G. A., Afek, Kreiss, Y. and Leshem, E. (2021). Early rate reductions of SARSCoV-2 infection and COVID-19 in BNT162b2 vaccine recipients. Lancet. DOI: 10.1016/S01406736(21)00448-7.

Andersen, K.G., Rambaut, A., Lipkin, W.I., Holmes, E.C., and Garry, R.F. (2020). The proximal origin of SARS-CoV-2. Nat. Med. DOI: 10.1038/s41591-0200820-9.

Andrade, V.M., Christensen-Quick, A., Agnes, J. , Tur, C., Reed, R. Kalia, I. Marrero, D. Elwood, K. Schultheis, M. Purwar, E. Reuschel, T. McMullan, P. Pezzoli, K. Kraynyak, A. Sylvester, M.P. Mammen, P. Tebas, J.J. Kim, D.B. Weiner, T.R.F. Smith, S.J. Ramos, L.M. Humeau, J.D. Boyer, and K.E. Broderick (2021). INO-4800 DNA Vaccine Induces Neutralizing Antibodies and T cell Activity Against Global SARSCoV-2 Variants. bioRxiv.

Anthony, S.J., R. Ojeda-Flores, O. Rico-Chavez, I. Navarrete-Macias, C.M. Zambrana-Torrelio, M.K. Rostal, J.H. Epstein, T. Tipps, E. Liang, M. SanchezLeon, J. Sotomayor-Bonilla, A.A. Aguirre, R. AvilaFlores, R.A. Medellin, T. Goldstein, G. Suzan, P. Daszak, and W.I. Lipkin, 2013: Coronaviruses in bats from Mexico. J Gen Viro 194, 1028-1038.

Baden, L.R., H.M. El Sahly, B. Essink, K. Kotloff, S. Frey, R. Novak, D. Diemert, S.A. Spector, N. Rouphael, C.B. Creech, J. McGettigan, S. Khetan, N. Segall, J. Solis, A. Brosz, C. Fierro, H. Schwartz, K. Neuzil, L. Corey, P. Gilbert, H. Janes, D. Follmann, M. Marovich, J. Mascola, L. Polakowski, J. Ledgerwood, B.S. Graham, H. Bennett, R. Pajon, C. Knightly, B. Leav, W. Deng, H. Zhou, S. Han, M. Ivarsson, J. Miller, and T. Zaks (2021). Efficacy and Safety of the mRNA-1273 SARS-CoV-2 Vaccine. N. Engl. J. Med. DOI: 10.1056/nejmoa2035389.

Banos, A., and J. Lacasa (2007). Spatio-temporal exploration of SARS epidemic. CyberGeo DOI: 10.4000/cybergeo.12803.

Cui, J., F. Li, and Z.L. Shi (2019). Origin and evolution of pathogenic coronaviruses. Nat. Rev. Microbiol. 17, 181-192, DOI: 10.1038/s41579-018-0118-9.

Dagan, N., N. Barda, E. Kepten, O. Miron, S. Perchik, M.A. Katz, M.A. Hernán, M. Lipsitch, B. Reis, and R.D. Balicer (2021). BNT162b2 mRNA Covid-19 Vaccine in a Nationwide Mass Vaccination Setting. N. Engl. J. Med. DOI: 10.1056/nejmoa2101765.
DIGESA, MSP, U. (2021). MSP, Estudio de efectividad de vacunación anti SARS-CoV-2 en Uruguay en 2021 [Online] Available at https://www.gub.uy/ ministerio-salud-publica/comunicacion/noticias/ segundo-estudio-efectividad-vacunacion-antisars-cov-2-uruguay-8-junio-2021.

Fields B. (2013). Virology Fields, 6th edn. Vol. 2 (Knipe D.M., \& Howley P.M., Eds.). Lippincott, Williams \& Wilkins.

Folegatti, P.M., K.J. Ewer, P.K. Aley, B. Angus, S. Becker, S. Belij-Rammerstorfer, D. Bellamy, S. Bibi, M. Bittaye, E.A. Clutterbuck, C. Dold, S.N. Faust, A. Finn, A.L. Flaxman, B. Hallis, P. Heath, D. Jenkin, R. Lazarus, R. Makinson, A.M. Minassian, K.M. Pollock, M. Ramasamy, H. Robinson, M. Snape, R. Tarrant, M. Voysey, C. Green, A.D. Douglas, A.V.S. Hill, T. Lambe, S.C. Gilbert, A.J. Pollard, J. Aboagye, K. Adams, A. Ali, E. Allen, J.L. Allison, R. Anslow, E.H. Arbe-Barnes, G. Babbage, K. Baillie, M. Baker, N. Baker, P. Baker, I. Baleanu, J. Ballaminut, E. Barnes, J. Barrett, L. Bates, A. Batten, K. Beadon, R. Beckley, E. Berrie, L. Berry, A. Beveridge, K.R. Bewley, E.M. Bijker, T. Bingham, L. Blackwell, C.L. Blundell, E. Bolam, E. Boland, N. Borthwick, T. Bower, A. Boyd, T. Brenner, P.D. Bright, C. BrownO'Sullivan, E. Brunt, J. Burbage, S. Burge, K.R. Buttigieg, N. Byard, I. Cabera Puig, A. Calvert, S. Camara, M. Cao, F. Cappuccini, M. Carr, M.W. Carroll, V. Carter, K. Cathie, R.J. Challis, S. Charlton, I. Chelysheva, J.-S. Cho, P. Cicconi, L. Cifuentes, H. Clark, E. Clark, T. Cole, R. Colin-Jones, C.P. Conlon, A. Cook, N.S. Coombes, R. Cooper, C.A. Cosgrove, K. Coy, W.E.M. Crocker, C.J. Cunningham, B.E. Damratoski, L. Dando, M.S. Datoo, H. Davies, H. De Graaf, T. Demissie, C. Di Maso, I. Dietrich, T. Dong, F.R. Donnellan, N. Douglas, C. Downing, J. Drake, R. Drake-Brockman, R.E. Drury, S.J. Dunachie, N.J. Edwards, F.D.L. Edwards, C.J. Edwards, S.C. Elias, M.J. Elmore, K.R.W. Emary, M.R. English, S. Fagerbrink, S. Felle, S. Feng, S. Field, C. Fixmer, C. Fletcher, K.J. Ford, J. Fowler, P. Fox, E. Francis, J. Frater, J. Furze, M. Fuskova, E. Galiza, D. Gbesemete, C. Gilbride, K. Godwin, G. Gorini, L. Goulston, C. Grabau, L. Gracie, Z. Gray, L.B. Guthrie, M. Hackett, S. Halwe, E. Hamilton, J. Hamlyn, B. Hanumunthadu, I. Harding, S.A. Harris, A. Harris, D. Harrison, C. Harrison, T.C. Hart, L. Haskell, S. Hawkins, I. Head, J.A. Henry, J. Hill, S.H.C. Hodgson, M.M. Hou, E. Howe, N. Howell, C. Hutlin, S. Ikram, C. Isitt, P. Iveson, S. Jackson, F. Jackson, S.W. James, M. Jenkins, E. Jones, K. Jones, C.E. Jones, B. Jones, R. Kailath, K. Karampatsas, J. 
Keen, S. Kelly, D. Kelly, D. Kerr, S. Kerridge, L. Khan, U. Khan, A. Killen, J. Kinch, T.B. King, L. King, J. King, L. Kingham-Page, P. Klenerman, F. Knapper, J.C. Knight, D. Knott, S. Koleva, A. Kupke, C.W. Larkworthy, J.P.J. Larwood, A. Laskey, A.M. Lawrie, A. Lee, K.Y. Ngan Lee, E.A. Lees, H. Legge, A. Lelliott, N.-M. Lemm, A.M. Lias, A. Linder, S. Lipworth, X. Liu, S. Liu, R. Lopez Ramon, M. Lwin, F. Mabesa, M. Madhavan, G. Mallett, K. Mansatta, I. Marcal, S. Marinou, E. Marlow, J.L. Marshall, J. Martin, J. McEwan, L. McInroy, G. Meddaugh, A.J. Mentzer, N. Mirtorabi, M. Moore, E. Moran, E. Morey, V. Morgan, S.J. Morris, H. Morrison, G. Morshead, R. Morter, Y.F. Mujadidi, J. Muller, T. Munera-Huertas, C. Munro, A. Munro, S. Murphy, V.J. Munster, P. Mweu, A. Noé, F.L. Nugent, E. Nuthall, K. O'Brien, D. O'Connor, B. Oguti, J.L. Oliver, C. Oliveira, P.J. O'Reilly, M. Osborn, P. Osborne, C. Owen, D. Owens, N. Owino, M. Pacurar, K. Parker, H. Parracho, M. Patrick-Smith, V. Payne, J. Pearce, Y. Peng, M.P. Peralta Alvarez, J. Perring, K. Pfafferott, D. Pipini, E. Plested, H. Pluess-Hall, K. Pollock, I. Poulton, L. Presland, S. Provstgaard-Morys, D. Pulido, K. Radia, F. Ramos Lopez, J. Rand, H. Ratcliffe, T. Rawlinson, S. Rhead, A. Riddell, A.J. Ritchie, H. Roberts, J. Robson, S. Roche, C. Rohde, C.S. Rollier, R. Romani, I. Rudiansyah, S. Saich, S. Sajjad, S. Salvador, L. Sanchez Riera, H. Sanders, K. Sanders, S. Sapaun, C. Sayce, E. Schofield, G. Screaton, B. Selby, C. Semple, H.R. Sharpe, I. Shaik, A. Shea, H. Shelton, S. Silk, L. Silva-Reyes, D.T. Skelly, H. Smee, C.C. Smith, D.J. Smith, R. Song, A.J. Spencer, E. Stafford, A. Steele, E. Stefanova, L. Stockdale, A. Szigeti, A. Tahiri-Alaoui, M. Tait, H. Talbot, R. Tanner, I.J. Taylor, V. Taylor, R. Te Water Naude, N. Thakur, Y. Themistocleous, A. Themistocleous, M. Thomas, T.M. Thomas, A. Thompson, S. Thomson-Hill, J. Tomlins, S. Tonks, J. Towner, N. Tran, J.A. Tree, A. Truby, K. Turkentine, C. Turner, N. Turner, S. Turner, T. Tuthill, M. Ulaszewska, R. Varughese, N. Van Doremalen, K. Veighey, M.K. Verheul, I. Vichos, E. Vitale, L. Walker, M.E.E. Watson, B. Welham, J. Wheat, C. White, R. White, A.T. Worth, D. Wright, S. Wright, X.L. Yao, and Y. Yau, (2020). Safety and immunogenicity of the ChAdOx1 $\mathrm{nCoV}-19$ vaccine against SARS-CoV-2: a preliminary report of a phase $1 / 2$, single-blind, randomised controlled trial. Lancet 396, 467-478, DOI: 10.1016/S01406736(20)31604-4.

Gorbalenya, A.E., L. Enjuanes, J. Ziebuhr, and E.J. Snijder (2006). Nidovirales: Evolving the largest
RNA virus genome. Virus Res. 117, 17-37, DOI: 10.1016/j.virusres.2006.01.017.

Keech, C., G. Albert, I. Cho, A. Robertson, P. Reed, S. Neal, J.S. Plested, M. Zhu, S. Cloney-Clark, H. Zhou, G. Smith, N. Patel, M.B. Frieman, R.E. Haupt, J. Logue, M. McGrath, S. Weston, P.A. Piedra, C. Desai, K. Callahan, M. Lewis, P. Price-Abbott, N. Formica, V. Shinde, L. Fries, J.D. Lickliter, P. Griffin, B. Wilkinson, and G.M. Glenn (2020). Phase 1-2 Trial of a SARS-CoV-2 Recombinant Spike Protein Nanoparticle Vaccine. N. Engl. J. Med. 383, DOI: 10.1056/nejmoa2026920.

Ksiazek, T.G., D. Erdman, C.S. Goldsmith, S.R. Zaki, T. Peret, S. Emery, S. Tong, C. Urbani, J.A. Comer, W. Lim, P.E. Rollin, S.F. Dowell, A.E. Ling, C.D. Humphrey, W.J. Shieh, J. Guarner, C.D. Paddock, P. Rota, B. Fields, J. DeRisi, J.Y. Yang, N. Cox, J.M. Hughes, J.W. LeDuc, W.J. Bellini, and L.J. Anderson (2003). A novel coronavirus associated with severe acute respiratory syndrome. N. Engl J. Med 348, 1953-1966.

Lau, S.K.P., P.C.Y. Woo, K.S.M. Li, Y. Huang, H.-W. Tsoi, B.H.L. Wong, S.S.Y. Wong, S.-Y. Leung, K.-H. Chan, and K.-Y. Yuen (2005). Severe acute respiratory syndrome coronavirus-like virus in Chinese horseshoe bats. Proc. Natl. Acad. Sci. U.S.A. DOI: 10.1073/pnas.0506735102.

Li, W., Z. Shi, M. Yu, W. Ren, C. Smith, J.H. Epstein, H. Wang, G. Crameri, Z. Hu, H. Zhang, J. Zhang, J. McEachern, H. Field, P. Daszak, B.T. Eaton, S. Zhang, and L.F. Wang, (2005). Bats are natural reservoirs of SARS-like coronaviruses. Science (80.).310, 676-679.

Logunov, D.Y., I. V. Dolzhikova, D. V. Shcheblyakov, A.I. Tukhvatulin, O. V. Zubkova, A.S. Dzharullaeva, A. V. Kovyrshina, N.L. Lubenets, D.M. Grousova, A.S. Erokhova, A.G. Botikov, F.M. Izhaeva, O. Popova, T.A. Ozharovskaya, I.B. Esmagambetov, I.A. Favorskaya, D.I. Zrelkin, D. V. Voronina, D.N. Shcherbinin, A.S. Semikhin, Y. V. Simakova, E.A. Tokarskaya, D.A. Egorova, M.M. Shmarov, N.A. Nikitenko, V.A. Gushchin, E.A. Smolyarchuk, S.K. Zyryanov, S. V. Borisevich, B.S. Naroditsky, and A.L. Gintsburg (2021). Safety and efficacy of an rAd26 and rAd5 vector-based heterologous primeboost COVID-19 vaccine: an interim analysis of a randomised controlled phase 3 trial in Russia. Lancet DOI: 10.1016/S0140-6736(21)00234-8. 
Maier, H.J., E. Bickerton, and P. Britton (2015). Coronaviruses: Methods and Protocols. Coronaviruses Methods Protoc.

Memish, Z.A., N. Mishra, K.J. Olival, S.F. Fagbo, V. Kapoor, J.H. Epstein, R. Alhakeem, A. Durosinloun, M. Al Asmari, A. Islam, A. Kapoor, T. Briese, P. Daszak, A.A. Al Rabeeah, and W.I. Lipkin (2013). Middle East respiratory syndrome coronavirus in bats, Saudi Arabia. Emerg Infect Dis19, 1819-1823.

Monto, A.S., P.M. DeJonge, A.P. Callear, L.A. Bazzi, S.B. Capriola, R.E. Malosh, E.T. Martin, and J.G. Petrie (2020). Coronavirus occurrence and transmission over 8 years in the HIVE cohort of households in Michigan. J. Infect. Dis. 222, DOI: 10.1093/infdis/ jiaa161.

Nalbandian, A., K. Sehgal, A. Gupta, M. V. Madhavan, C. McGroder, J.S. Stevens, J.R. Cook, A.S. Nordvig, D. Shalev, T.S. Sehrawat, N. Ahluwalia, B. Bikdeli, D. Dietz, C. Der-Nigoghossian, N. Liyanage-Don, G.F. Rosner, E.J. Bernstein, S. Mohan, A.A. Beckley, D.S. Seres, T.K. Choueiri, N. Uriel, J.C. Ausiello, D. Accili, D.E. Freedberg, M. Baldwin, A. Schwartz, D. Brodie, C.K. Garcia, M.S.V. Elkind, J.M. Connors, J.P. Bilezikian, D.W. Landry, and E.Y. Wan, (2021). Postacute COVID-19 syndrome. Nat. Med. 27, DOI: 10.1038/s41591-021-01283-z.

Naveca, F., C. da Costa, V. Nascimento, V. Souza, A. Corado, Á. Nascimento, FernandaCosta, D. Duarte, G. Silga, M. Mejía, K. Pessoa, L. Gonçalves, M.J. Brandão, M. Jesus, R. Pinto, M. Silva, T. Mattos, L. Abdalla, J.H. Santos, R. Costa-Filho, G.L. Walllau, M. Mendonça Siqueira, N. Delatorre, T. Gräf, G. Bello, and P.C. Resende (2021). SARS-CoV-2 reinfection by the new Variant of Concern (VOC) P.1 in Amazonas, Brazil. Virological.Org.

Palacios, R., A.P. Batista, C.S.N. Albuquerque, E.G. Patiño, J. do P. Santos, M. Tilli Reis Pessoa Conde, R. de O. Piorelli, L.C. Pereira Júnior, S.M. Raboni, F. Ramos, G.A. Sierra Romero, F.E. Leal, L.F.A. Camargo, F.H. Aoki, E.B. Coelho, D.S. Oliveira, C.J.F. Fontes, G.C.S. Pileggi, A.L.L. de Oliveira, A.M. de Siqueira, D.B.L. de Oliveira, V.F. Botosso, G. Zeng, Q. Xin, M.M. Teixeira, M.L. Nogueira, and E.G. Kallas (2021). Efficacy and Safety of a COVID-19 Inactivated Vaccine in Healthcare Professionals in Brazil: The PROFISCOV Study. SSRN Electron. J. DOI: $10.2139 /$ ssrn. 3822780 .

Polack, F.P., S.J. Thomas, N. Kitchin, J. Absalon, A. Gurtman, S. Lockhart, J.L. Perez, G. Pérez Marc, E.D. Moreira, C. Zerbini, R. Bailey, K.A. Swanson,
S. Roychoudhury, K. Koury, P. Li, W. V. Kalina, D. Cooper, R.W. Frenck, L.L. Hammitt, Ö. Türeci, H. Nell, A. Schaefer, S. Ünal, D.B. Tresnan, S. Mather, P.R. Dormitzer, U. Şahin, K.U. Jansen, and W.C. Gruber (2020). Safety and Efficacy of the BNT162b2 mRNA Covid-19 Vaccine. N. Engl. J. Med. DOI: 10.1056/nejmoa2034577.

Sadoff, J., M. Le Gars, G. Shukarev, D. Heerwegh, C. Truyers, A.M. de Groot, J. Stoop, S. Tete, W. Van Damme, I. Leroux-Roels, P.-J. Berghmans, M. Kimmel, P. Van Damme, J. de Hoon, W. Smith, K.E. Stephenson, S.C. De Rosa, K.W. Cohen, M.J. McElrath, E. Cormier, G. Scheper, D.H. Barouch, J. Hendriks, F. Struyf, M. Douoguih, J. Van Hoof, and H. Schuitemaker (2021) Interim Results of a Phase 1-2a Trial of Ad26.COV2.S Covid-19 Vaccine. N. Engl. J. Med. DOI: 10.1056/nejmoa2034201.

Smith, I., and L.F. Wang (2013). Bats and their virome: An important source of emerging viruses capable of infecting humans. Curr. Opin. Virol. 3, 84-91, DOI: 10.1016/j.coviro.2012.11.006.

Song, Z., Y. Xu, L. Bao, L. Zhang, P. Yu, Y. Qu, H. Zhu, W. Zhao, Y. Han, and C. Qin (2019). From SARS to MERS, Thrusting Coronaviruses into the Spotlight. Viruses 11, 59, DOI: 10.3390/v11010059.

Wang, N., S.Y. Li, X. Lou Yang, H.M. Huang, Y.J. Zhang, H. Guo, C.M. Luo, M. Miller, G. Zhu, A.A. Chmura, E. Hagan, J.H. Zhou, Y.Z. Zhang, L.F. Wang, P. Daszak, and Z.L. Shi (2018). Serological Evidence of Bat SARS-Related Coronavirus Infection in Humans, China. Virol. Sin. DOI: 10.1007/s12250-018-0012-7.

Xia, S., Y. Zhang, Y. Wang, H. Wang, Y. Yang, G.F. Gao, W. Tan, G. Wu, M. Xu, Z. Lou, W. Huang, W. Xu, B. Huang, H. Wang, W. Wang, W. Zhang, N. Li, Z. Xie, L. Ding, W. You, Y. Zhao, X. Yang, Y. Liu, Q. Wang, L. Huang, Y. Yang, G. Xu, B. Luo, W. Wang, P. Liu, W. Guo, and X. Yang (2021). Safety and immunogenicity of an inactivated SARS-CoV-2 vaccine, BBIBP-CorV: a randomised, double-blind, placebo-controlled, phase 1/2 trial. Lancet Infect. Dis. DOI: 10.1016/S1473-3099(20)30831-8.

Xie, X., Y. Liu, J. Liu, X. Zhang, J. Zou, C.R. FontesGarfias, H. Xia, K.A. Swanson, M. Cutler, D. Cooper, V.D. Menachery, S.C. Weaver, P.R. Dormitzer, and P.Y. Shi (2021). Neutralization of SARS-CoV-2 spike $69 / 70$ deletion, E484K and N501Y variants by BNT162b2 vaccine-elicited sera. Nat. Med. 27, DOI: 10.1038/s41591-021-01270-4. 
Zhang, Y., G. Zeng, H. Pan, C. Li, Y. Hu, K. Chu, W. Han, Z. Chen, R. Tang, W. Yin, X. Chen, Y. Hu, X. Liu, C. Jiang, J. Li, M. Yang, Y. Song, X. Wang, Q. Gao, and F. Zhu (2021). Safety, tolerability, and immunogenicity of an inactivated SARS-CoV-2 vaccine in healthy adults aged 18-59 years: a randomised, doubleblind, placebo-controlled, phase $1 / 2$ clinical trial. Lancet Infect. Dis. 21, DOI: 10.1016/S14733099(20)30843-4. 